\title{
A Jet-ADAF model for Sgr A*
}

\author{
F. Yuan`, S. Markoff ${ }^{\star \star}$, and H. Falcke \\ Max-Planck-Institut für Radioastronomie, Auf dem Hügel 69, 53121 Bonn, Germany
}

Received 5 July 2001 / Accepted 3 December 2001

\begin{abstract}
The recent Chandra observation of the radio source at the center of our Galaxy, Sgr A*, puts new constraints on its theoretical models. The spectrum is very soft, and the source is rapidly variable. We consider different models to explain the observations. We find that the features of the X-ray spectrum can be marginally explained with an advection-dominated accretion flow (ADAF) model while it does not well fit the radio spectrum. An ADAF with strong winds (ADIOS) model is not favored if we assume that the wind does not radiate. Alternatively, we propose a coupled jet plus accretion disk model to explain the observations for Sgr A*. The accretion flow is described as an ADAF fed by Bondi-Hoyle accretion of hot plasma in the Galactic Center region. A small fraction of the accretion flow is ejected near the black hole, forming a jet after passing through a shock. As a result, the electron temperature increases to $\sim 2 \times 10^{11} \mathrm{~K}$, which is about 10 times higher than the highest temperature attained in the ADAF. The model is self-consistent since the main jet parameters are determined by the underlying accretion disk at the inner edge. The emergent spectrum of $\mathrm{Sgr} \mathrm{A}^{*}$ is the sum of the emission from jet and underlying ADAF. The very strong Comptonization of synchrotron emission from the jet can dominate the bremsstrahlung from the ADAF, therefore, a very short variability timescale is expected and the predicted $\mathrm{X}$-ray slope and the radio spectrum is in very good agreement with the observations.
\end{abstract}

Key words. accretion, accretion disks - black hole physics - galaxies: active - galaxies: nuclei - Galaxy: center hydrodynamics

\section{Introduction}

The energetic radio source $\operatorname{Sgr} \mathrm{A}^{*}$ located at the center of our Galaxy is now widely believed to be the signature of a massive black hole with mass $M=2.6 \times 10^{6} M_{\odot}$ (Melia \& Falcke 2001; Haller et al. 1996; Eckart \& Genzel 1996; Ghez et al. 1998; Reid et al. 1999; Backer \& Sramek 1999). Its radio spectrum seems to consist of two components, with a break around $\sim 50 \mathrm{GHz}$. The spectral dependence is $F_{\nu} \propto \nu^{0.2}$ for $\nu<50 \mathrm{GHz}$, while above this break there is a submm bump which is described by $F_{\nu} \propto \nu^{0.8}$ up to $\sim 10^{3} \mathrm{GHz}$ followed by a steep cut-off towards the infrared (IR) (Zylka et al. 1992; Serabyn et al. 1997; Falcke et al. 1998). The upper limits from IR (Menten et al. 1997) and ROSAT X-ray observations (Predehl \& Trümper 1994) indicate that this source is quite dim.

On the theoretical side, a number of models have been proposed in the past years for Sgr A* Most models are based on accretion onto the central massive black hole.

\footnotetext{
Send offprint requests to: F. Yuan, e-mail: fyuan@mpifr-bonn.mpg.de

* Also at CAS-PKU Joint Beijing Astrophysical Centre, Beijing 100871, China; and Astronomical and Astrophysical Center of East China, Nanjing University, Nanjing 210093, China.

$\star \star$ Humboldt research fellow.
}

Possible sources of accretion material include the stellar winds emitted by the nearby massive stars and the hot interstellar medium. Since in either case the angular momentum of the accretion flow should be small, Melia $(1992,1994)$ proposed a spherical accretion model. In this model the accretion flow is assumed to free-fall until a Keplerian disk is formed within a small "circularization" radius. The main contributors to the radio and X-ray spectra are synchrotron radiation and bremsstrahlung, respectively, from the roughly free-fall flow beyond the small disk. However, spherical accretion is likely to be an oversimplification, since the accretion flow still possesses some angular momentum. An advection-dominated accretion flow (ADAF) model therefore is more dynamically exact in this sense (Narayan et al. 1995; Manmoto et al. 1997; Narayan et al. 1998). The most attractive feature of the ADAF model is its ability to explain the unusual lowluminosity of $\mathrm{Sgr} \mathrm{A}^{*}$ given the relatively abundant accretion material. This is because most of the viscously dissipated energy is stored in the flow and advected beyond the event horizon rather than radiated away (Ichimaru 1977; Rees et al. 1982; Narayan \& Yi 1994, 1995; Abramowicz et al. 1995; Chen et al. 1995; Narayan et al. 1997; Chen et al. 1997). In the application to Sgr A*, the radio spectrum is produced by the synchrotron process in the innermost region of the disk while the X-rays are due to 
bremsstrahlung radiation of the thermal electrons in a large range of radii $\sim 10^{3}-10^{4} R_{\mathrm{s}}$, where $R_{\mathrm{s}}=2 \mathrm{GM} / \mathrm{c}^{2}$ is the Schwarzschild radius. However, the ADAF underpredicts the low-frequency radio emission of Sgr A* by over an order of magnitude and additional assumptions must be imposed in order to match the spectrum (Mahadevan 1998; Özel et al. 2000).

Following the initial paper by Reynolds \& McKee (1980) (see also Blandford \& Königl 1979), Falcke et al. (1993) proposed that it is the jet stemming from the disk rather than the disk itself which is responsible for the radio spectrum of Sgr A*. In this model, the submm bump is produced by the acceleration zone of the jet, called nozzle, while the low-frequency radio spectrum comes from the part of the jet beyond the nozzle (Falcke 1996b; Falcke \& Biermann 1999). The nozzle is of order $10 R_{\mathrm{S}}$ and forms from the disk at a radius of $\sim 2 R_{\mathrm{s}}$. This model gives an excellent fit to the radio spectrum of $\mathrm{Sgr} \mathrm{A}^{*}$, including the low-frequency spectrum below the break and the submm bump, but the expected X-ray emission was not calculated explicitly.

The latest observational constraints for Sgr A* come from the high spatial resolution $\left(\approx 1^{\prime \prime}\right)$ Chandra X-ray Observatory (Baganoff et al. 2001a, 2001b). Baganoff et al. observed Sgr A* twice and they found that Sgr A* comes in two states: quiescent and flares. In the present paper we concentrate on the quiescent state, whereas the flare state is considered in Markoff et al. (2001b). The main observational results for the quiescent state are summarized as follows ${ }^{1}$ :

- The absorption-corrected $2-10 \mathrm{keV}$ luminosity is $\left(2.2_{-0.2}^{+0.4}\right) \times 10^{33} \mathrm{erg} \mathrm{s}^{-1}$;

- The spectrum is well fitted by an absorbed power-law model with photon index $\Gamma=2.2_{-0.7}^{+0.5}$;

- The inner region of the source is rapidly variable on short timescale of $\simeq 1 \mathrm{hr}$. A rapid drop of flux on a timescale of $10 \mathrm{~min}$ is detected in the flare state. On the other hand, the comparison between the two observations with an interval of about one year indicates that the steady X-ray flux remains almost constant;

- Some fraction of the X-ray flux may come from a partly extended region with diameter $\approx 1^{\prime \prime}$;

- There is tentative evidence for a $\mathrm{Fe} \mathrm{K} \alpha$ line at $6.7 \mathrm{keV}$.

These results provide new and strict constraints to the theoretical models for Sgr A*. In both the ADAF and spherical accretion models mentioned above, the X-ray radiation is produced by bremsstrahlung originating from $10^{3}-10^{4} R_{\mathrm{s}}$. Hence the spectrum is very hard with photon index $\Gamma \sim 1.4$ and the predicted variability timescale is thousands of hours, much longer than the observed $\sim 1$ hour.

1 The luminosity and especially the photon index are taken from Baganoff et al. (2001b), which are slightly different from those in Baganoff et al. (2001a) where the spectral models used did not account for dust scattering; see Baganoff et al. (2001b) for details.
Therefore it is necessary to reexamine the theoretical models for Sgr A*. Melia et al. (2001) proposed that the electrons in the small Keplerian disk can attain a very high temperature through some magnetic processes, and the resulting synchrotron and self-Compton emission are responsible for the radio and X-ray spectrum. However, the formation of the small disk may not be a necessary result of such low angular momentum accretion. An accretion flow with very low angular momentum can still be described by an ADAF, although such accretion may belong to the Bondi-like type rather than disk-like type, as shown by Yuan (1999) (see also Abramowicz \& Zurek 1981; Abramowicz 1998). Thus the dynamical scenario of this model needs to be studied carefully.

For the jet model, Falcke \& Markoff (2000) take into account the contribution from synchrotron self-Compton emission (SSC) in the nozzle and find that the parameters required to interpret the submm bump give a very good fit to the Chandra spectrum without changing the basic parameters of the jet model. But the remaining important problem in the model is why the parameters of the jet possess the required values, particularly in reference to the inferred underlying accretion disk. Previous ideas of a standard optically thick accretion disk in Sgr A* (e.g., Falcke \& Heinrich 1994) do not seem to work because the predicted IR flux from a standard thin disk with a reasonable accretion rate would be several orders of magnitude higher than the observed IR upper limit (Falcke \& Melia 1997). Therefore, it is crucial to consider the jet and accretion flow as a coupled system in Sgr A*, and to consider what are their respective roles if both are truly present in Sgr A*. Yuan (2000) presented the first effort, by considering a combination of jet and ADAF models. However, the complete Chandra data was not available at that time and the detailed coupling mechanism was lacking in Yuan (2000) so it is necessary to revisit the model again.

The development of the theory provides a new chance to model Sgr A*. Since the Bernoulli parameter of the ADAF is positive, which means the gas can escape to infinity with positive energy, Blandford \& Begelman (1999) propose an advection-dominated inflow-outflow solution (ADIOS) in which most of the gas is lost through winds rather than accreted past the horizon of the black hole. The concept of strong winds from accretion flow was also proposed and studied by Xu \& Chen (1997) and Das \& Chakrabarti (1999). The latter described pressuredriven winds from centrifugally supported boundary layers and shocks in the inner regions of disks, and the former proposed an advection-dominated flow where the central black hole redirects the inward flow at low latitudes into an outflow at high latitudes. We are not explicitly making use of the latter two models. The most appealing point of the ADIOS model as applied to $\mathrm{Sgr} \mathrm{A}^{*}$ is that the predicted X-ray spectrum is possibly much softer than that of the ADAF (Quataert \& Narayan 1999), and therefore could possibly give a better fit to the Chandra data. This is because the density profile of the accretion flow becomes flatter due to the wind, while X-ray emission at higher 
frequencies is produced in the inner region of the accretion flow. If we assume that the mass accretion rate in the ADIOS is described by a power-law of radius, $\dot{M} \propto R^{p}$, the predicted photon index in Chandra band is approximately $\Gamma \approx 3 / 2+2 p$. Thus it is necessary to investigate this model for the possibility of interpreting the Chandra results.

In this paper we explore several of the abovementioned models for Sgr A*. By probing a larger parameter space than before, we find that ADAFs can give a marginal interpretation to the new Chandra results, although the fit is not very good in some points (Sect. 2), while the ADIOS model can't (Sect. 3). In Sect. 4 we propose that the combination of an ADAF and a jet could provide an excellent fit to the observations to $\mathrm{Sgr} \mathrm{A}^{*}$, and present our model results. The last section is a summary and discussion.

\section{ADAFs}

We first model Sgr A* with the advection-dominated accretion model. The modeling technique is described in detail in Yuan et al. (2000; see also Nakamura et al. 1997). We use the Paczyński \& Wiita (1980) potential to mimic the geometry of the central black hole. A randomly oriented magnetic field is assumed to exist in the accretion flow and the ratio between the gas pressure and total pressure (gas pressure plus magnetic pressure) is denoted as $\beta$. As commonly used, we assume that a fraction, $\delta$, of viscous dissipation will directly heat electrons. The radiation mechanisms we consider include bremsstrahlung, synchrotron radiation and their Comptonization. We require a physical global solution that satisfies the no-torque condition at the horizon of the black hole, a sonic point condition, and the outer boundary conditions. The calculation of the spectra and the structure of the accretion flows are made completely self-consistent as the full set of coupled radiation hydrodynamical accretion equations are solved numerically.

The parameters are adopted as follows. We take the black hole mass as $M=2.5 \times 10^{6} M_{\odot}$, and the viscosity parameter is fixed as $\alpha=0.1$. We assume the magnetic field is in equipartition with the gas pressure or weaker, i.e., $\beta=0.5,0.9,0.99$, although the sub-equipartition magnetic field is more plausible if $\alpha=0.1$. We set $\delta$ as $10^{-3}$ or $10^{-2}$ as usual, i.e., we assume that most of the viscous dissipation will heat ions. For the mass accretion rate, using their latest Chandra observational data, Baganoff et al. (2001a) estimate $\dot{M} \sim 3 \times 10^{-6} M_{\odot} \mathrm{yr}^{-1}$, if the stellar wind is the accretion material, or $\dot{M} \sim 1 \times 10^{-6} M_{\odot} \mathrm{yr}^{-1}$, if the hot ISM around Sgr $\mathrm{A}^{*}$ serves as the accretion source, which we use as our reference numbers. In principle, the accretion rate of $\mathrm{Sgr} \mathrm{A}^{*}$ could be much higher, based on the available material from stellar winds. However, explosive events like the hyper-/supernova Sgr A East could temporarily reduce the accretion rate onto $\mathrm{Sgr} \mathrm{A}^{*}$ substantially (e.g., Coker 2001).

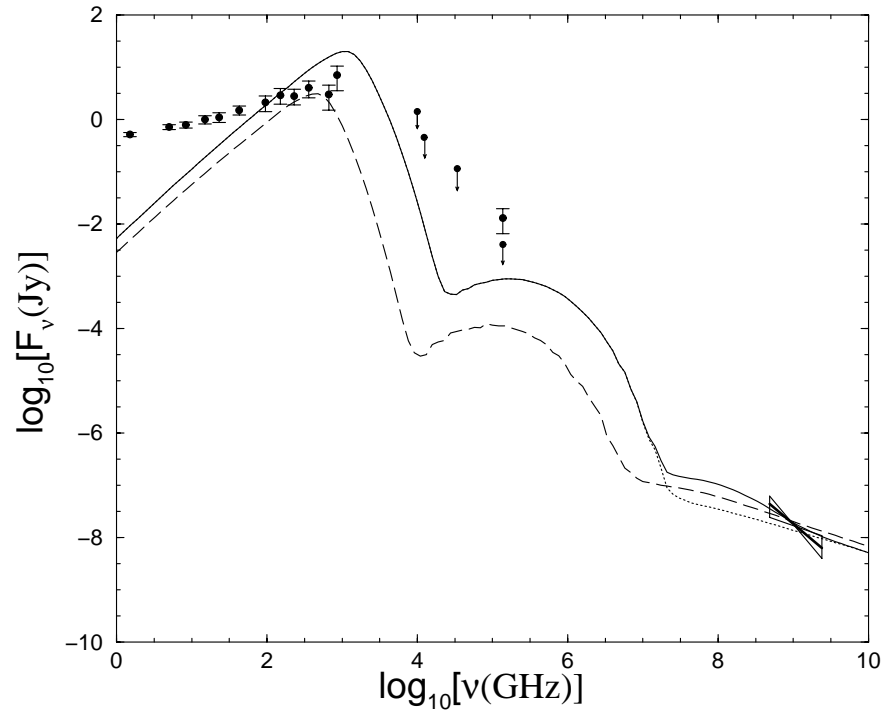

Fig. 1. Two fits for a standard ADAF model of Sgr A*. The radio and IR data are compiled by Melia \& Falcke (2001). The short solid line in the X-ray error box shows the best fit to the Chandra observation by a power-law model in Baganoff et al. (2001b). The parameters for the solid line are $\dot{M}=2.8 \times$ $10^{-5} \dot{M}_{\mathrm{Edd}}, \alpha=0.1, \beta=0.9, \delta=10^{-2}$. For the dashed line, $\dot{M}=3 \times 10^{-5} \dot{M}_{\mathrm{Edd}}, \alpha=0.1, \beta=0.99, \delta=10^{-3}$. The outer boundary conditions are $T_{\mathrm{i}} \approx T_{\mathrm{e}}=8 \times 10^{6} \mathrm{~K}$ (both), $\Omega_{\text {out }}=$ $0.16 \Omega_{\text {Kepler }}$ (solid line) and $\Omega_{\text {out }}=0.15 \Omega_{\text {Kepler }}$ (dashed line).

The outer boundary conditions should be taken seriously since they may affect the emergent spectrum significantly (Yuan et al. 2000). Throughout this paper we set the outer boundary of the accretion flow at $R_{\text {out }}=10^{5} R_{\mathrm{s}}$, where $R_{\mathrm{S}}$ is the Schwarzschild radius of the black hole, since this is approximately the location where the accretion begins according to the Bondi-Hoyle capture theory. Three outer boundary conditions are the temperatures of ions and electrons, $T_{\mathrm{i}, \mathrm{e}}$, and the angular velocity of the accretion flow, $\Omega_{\text {out }}$, at $R_{\text {out }}$. When $R_{\text {out }}$ is very large, as in the present case, the available range of $T_{\mathrm{i}, \mathrm{e}}$ within which we can get a physical solution is small, therefore the effect of $T_{\mathrm{i}, \mathrm{e}}$ can be neglected. But the feasible range of $\Omega_{\text {out }}$ is large and may have a significant effect on the emergent spectrum. For fixed parameters $R_{\text {out }}, \beta$ and $\alpha$, there exists a critical value of $\Omega_{\text {out }}$ above which the accretion is of disk-like type while below it is of Bondi-like type (Yuan 1999). The density of the Bondi-like accretion flow is much lower than the disk-like type at the same mass accretion rate. Unfortunately the exact value of $\Omega_{\text {out }}$ is uncertain. We only know that it must be low no matter whether it originates from stellar winds or from the hot ISM. For example, the hydrodynamical simulations of Coker \& Melia (1997) found $\Omega_{\text {out }} \sim 0.2 \Omega_{\text {Kepler }}$, if it comes from stellar winds. We therefore require in our model that $\Omega_{\text {out }} \lesssim 0.3 \Omega_{\text {Kepler }}$.

Figure 1 shows our fits to the spectrum with an ADAF model. The radio and IR data are compiled by Melia \& Falcke (2001). The short solid line within the error box shows the best fit to the Chandra observation by a 
power-law model in Baganoff et al. (2001b). We show $F_{\nu}$ rather than $\nu F_{\nu}$ because it is more conducive for judging the quality of the spectral fit at radio bands. The solid line shows our best fit to the spectrum especially to the Chandra data. The parameters are $\alpha=0.1, \beta=0.9, \delta=$ $10^{-2}$, and $\dot{M}=1.55 \times 10^{-6} M_{\odot} \mathrm{yr}^{-1}$ (or $2.8 \times 10^{-5} \dot{M}_{\mathrm{Edd}}$, here the Eddington accretion rate is defined as $\dot{M}_{\text {Edd }}=$ $L_{\text {Edd }} / 0.1 c^{2}=5.525 \times 10^{-2} M_{\odot} \mathrm{yr}^{-1}$ where $L_{\mathrm{Edd}}$ is the Eddington luminosity). The outer boundary condition at $R_{\text {out }}=10^{5} R_{\mathrm{s}}$ is $T_{\mathrm{i}} \approx T_{\mathrm{e}}=8 \times 10^{6} \mathrm{~K}$ (this value of temperature is consistent with the X-ray observations by Baganoff et al. 2001a), $\Omega_{\text {out }}=0.16 \Omega_{\text {Kepler }}$.

From the figure we find that an ADAF model can fit the X-ray spectrum with a reasonable accretion rate $^{2}$ although the predicted spectrum is flatter than the best fit of Baganoff et al. (2001b). The predicted X-ray spectrum is composed of two components, namely the bremsstrahlung from the outer region of ADAF and the second-order SSC from the innermost region of ADAF. The dotted line in the figure shows the result excluding the SSC component. Different from our result, in the ADAF model of Narayan et al. (1998), the X-ray emission is dominated by bremsstrahlung alone. One reason for the difference is our use of a higher $\delta$, and another reason is that we treat the outer boundary conditions more carefully. For bremsstrahlung, the emission at a frequency $\nu$ is dominated by the largest radius in an ADAF that satisfies $h \nu \sim k T(r)$. Our numerical calculation results indicate that the 2 and $10 \mathrm{keV}$ radiation is dominated by radii around $4 \times 10^{4} R_{\mathrm{s}}$ and $7400 R_{\mathrm{s}}$, respectively. This large radial range is consistent with the extended emission component $\left(\approx 1^{\prime \prime} \approx 10^{5} R_{\mathrm{s}}\right.$ ) observed by Chandra. In addition, the thermal bremsstrahlung can also explain the possible $\mathrm{Fe} \mathrm{K} \alpha$ emission line at $6.7 \mathrm{keV}$ (Narayan \& Raymond 1999). The dynamical timescale of the accretion flow at these large radii, which is responsible for the bremsstrahlung variability, is $t_{\mathrm{d}} \approx\left(R^{3} / G M\right)^{1 / 2} \sim$ 1 year. Baganoff et al. (2001b) made a comparison between their two observations with an interval of about one year and found that the steady state X-ray flux remains almost constant. This result, combined with the rapid variability, seems to indicate that there are two components to the X-ray emission operating on very different spatial scales and having very different time scales for variability. Bremsstrahlung may well be the component responsible for the constant flux. The SSC component mainly comes from regions $\lesssim 3 R_{\mathrm{s}}$ (see Fig. 1 in Manmoto et al. 1997). The corresponding variability timescale is $\sim 3 R_{\mathrm{s}} / v_{\mathrm{r}} \approx 1000 \mathrm{~s}$. So this component would be responsible for the observed rapid variability.

\footnotetext{
${ }^{2}$ In Yuan (2000), the Chandra flux is produced with a higher accretion rate, $1.5 \times 10^{-4} \dot{M}_{\text {Edd }}$. The discrepancy in accretion rate is because in Yuan (2000) the accretion is Bondi-like, while in the present paper it is disk-like. For the same accretion rate, the density in a Bondi-like accretion flow is much lower than in a disk-like flow. Since bremsstrahlung emission is proportional to the square of the density the X-ray emission can be largely different between the two cases.
}

However, as shown by Fig. 1, this model over-predicts the submm bump by a factor of $\sim 2-3$. We then try to lower the synchrotron flux from the ADAF to fit the submm bump better, as shown by the dashed line in Fig. 1. The parameters are $\dot{M}=1.66 \times 10^{-6} M_{\odot} \mathrm{yr}^{-1}, \beta=0.99, \delta=$ $10^{-3}$. The outer boundary conditions are the same as the solid line except with $\Omega_{\text {out }}=0.15 \Omega_{\text {Kepler }}$. In this case the second order SSC will become too weak to contribute to the X-ray flux, therefore, bremsstrahlung is almost the sole contributor to the X-ray spectrum. Consequently, the predicted spectrum is too flat and the $\sim 1$ hour variability is hard to explain. Considering that we can only investigate a limited parameter space of the ADAF model, and the fact that the solid line only fits marginally, we conclude that it is possible to interpret the spectrum of $\mathrm{Sgr} \mathrm{A}$ * from submm bump to X-ray using the ADAF model. However, it remains to be seen whether the current ADAF model can indeed produce a strong flare as found by Baganoff et al. (2001b). In addition, as in all previous ADAF models in the literature, the ADAF model always under-predicts the low-frequency radio spectrum which needs a contribution from another component such as a jet.

\section{ADIOS}

We next attempt to model Sgr A* with an ADIOS. The modeling approach is exactly the same as with the ADAF, except that the accretion rate is assumed to be described by $\dot{M}=\dot{M}_{0}\left(R / R_{\text {out }}\right)^{p}$. We solve the full set of coupled radiation hydrodynamical accretion equations to obtain the spectra and the structures of the accretion flow consistently. Note that this is an improvement compared to Quataert \& Narayan (1999) where some dynamical quantities such as radial velocity and sound speed obtained in corresponding ADAFs (with $\dot{M}=\dot{M}_{0}$ ) are used in calculating the spectra of the ADIOS. Following Quataert \& Narayan (1999), we assume that the wind does not radiate.

We first assume that the fraction of viscous heating of electrons is $\delta=10^{-3}$. We set $\alpha=0.1$ but treat $\dot{M}, p$ and $\beta$ as free in order to find the best set of parameters to fit the submm bump and the X-ray spectrum. The dashed line in Fig. 2 shows our best model results. The parameters are $\dot{M}_{0}=1.66 \times 10^{-5} M_{\odot} \mathrm{yr}^{-1}, p=0.28, \alpha=0.1$, but $\beta=0.5$ (not 0.9 since otherwise the predicted radio flux is too low compared to the observation). The outer boundary conditions are $T_{\mathrm{i}} \approx T_{\mathrm{e}}=8 \times 10^{6} \mathrm{~K}$, and $\Omega_{\text {out }}=0.295 \Omega_{\text {Kepler }}$ at $R_{\text {out }}=10^{5} R_{\mathrm{s}}$. Compared to the ADAF model, both the slope of the X-ray spectrum and the submm bump are now fitted better. However, there are two serious problems for this fit. The first one is that the required mass accretion rate is over 5 times higher than the upper limit estimated in Baganoff et al. (2001a) mentioned above. The second problem is that the X-ray spectra are produced by thermal bremsstrahlung emission alone, therefore this model cannot explain the short timescale variability. In fact, the introduction of a wind makes the variability timescale even longer because the decreasing density of accretion flows (e.g. Di Matteo et al. 2000) makes things worse. 


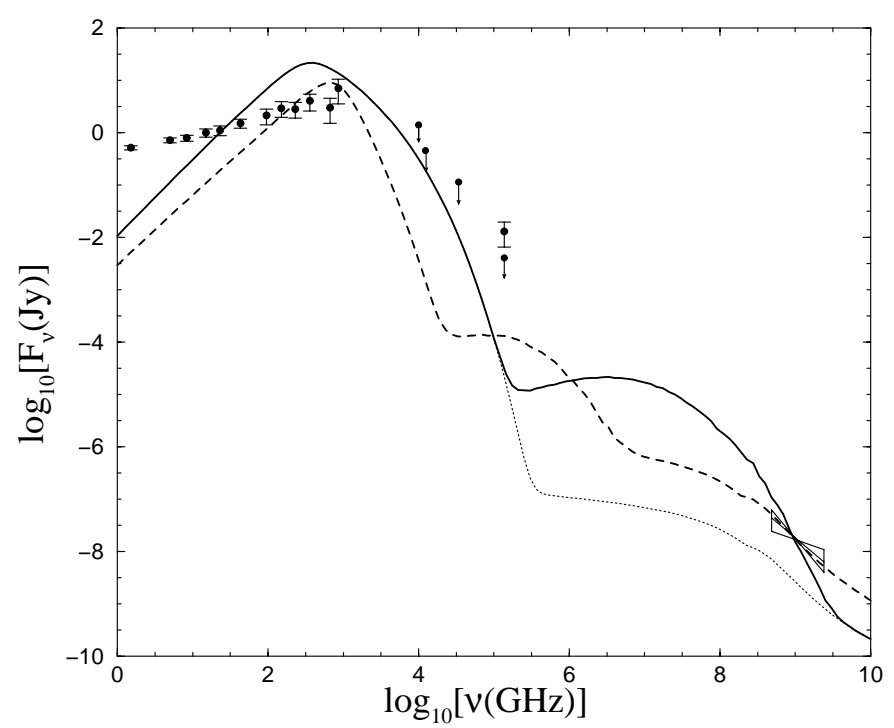

Fig. 2. Three ADIOS spectral models for Sgr A*. The shortdashed line is for $\delta=10^{-3}, p=0.28$, the long-dashed line for $\delta=10^{-3}, p=0.6$. The solid line is for $\delta=1, p=0.4$, the dotted line is exactly the same model as the solid line, except that Comptonization of synchrotron radiation is neglected. See text for other parameters.

The very rapid variability observed by Chandra indicates that the X-ray emission comes from a very small spatial region. This points towards SSC occurring in the inner region of the disk. In the case of the existence of strong winds, the density of the accretion flow in the innermost region is very low. When the flow is tenuous, SSC will show some spectral peaks as a result of different scattering orders. To make SSC dominate over bremsstrahlung in the X-ray band, the first order of SSC is more promising due to the rapid decrease of Compton scattering probability with increasing scattering orders. To make the first order SSC component reach the Chandra band, the electron temperature in the emission region must be very high.

An effective way to increase the electron temperature in the accretion flow is to increase $\delta$. In the ADAF we generally assume that $\delta$ is as as small as $\delta=10^{-3}$ or $10^{-2}$, i.e., the viscous dissipation mainly heats the ions. However, because of the uncertainty in the microphysics of the ADAF, it is possible that for some reasons, such as magnetic reconnection, the viscous dissipation may prefer heating electrons, i.e., $\delta$ may be much larger (Bisnovatyi-Kogan \& Lovelace 1997, 2000; Gruzinov 1998; Quataert \& Gruzinov 1999; Blackman 1999). In this case, the temperature of the electrons will be greatly increased.

We try to model the spectrum using various values for $\delta$. We find that only when $\delta \approx 1$, i.e., almost all of the viscous dissipation heats only electrons, can we get a high enough electron temperature to make the first order SSC dominate the X-ray emission. The solid line in Fig. 2 shows such an example. Other parameters in this model are $\dot{M}_{0}=2.8 \times 10^{-6} M_{\odot} \mathrm{yr}^{-1}, \alpha=0.1, \beta=0.9$, and $p=0.4$. The outer boundary conditions are $T_{\mathrm{i}, \mathrm{e}}=10^{7} \mathrm{~K}$, $\Omega_{\text {out }}=0.25 \Omega_{\text {Kepler }}$. The temperature of electrons is as high as $10^{11} \mathrm{~K}$ for the accretion flow within $6 R_{\mathrm{s}}$ and the highest temperature is $3 \times 10^{11} \mathrm{~K}$. This model is then very similar to the model proposed by Melia et al. (2001) for Sgr A* in the sense that a high-temperature inner disk forms, with $T_{\mathrm{e}}>10^{11} \mathrm{~K}$. Synchrotron emission in this hottest region produces the submm bump, synchrotron self-Compton dominates the X-ray band and gives a very soft spectrum. The thermal bremsstrahlung radiation only contributes a small part as shown by the dotted line, where $\mathrm{SSC}$ is neglected. In this case a very short X-ray variability timescale can be expected.

Putting aside the reality of such a high $\delta$, the fit is not satisfactory on the following points: first, it underpredicts the low-frequency radio spectrum. Second, the predicted X-ray slope is much steeper than the best fit of Baganoff et al. (2001b). The third problem is that this model over-predicts the flux above $\sim 100 \mathrm{GHz}$ by a factor of 4-6. We cannot get a better fit no matter how we adjust the parameters. Because of the strong self-absorption of synchrotron emission, the radio spectrum is the result of a super-position of blackbody radiation from the different parts of the ADAF with different temperatures. So, comparing this model with an ADAF (or ADIOS with small $\delta$ ), we can understand that the main reason for the over-prediction is its too extreme temperature making the flux of the blackbody radiation stronger. Thus we conclude that, if we do not consider the possible radiation of winds, the ADIOS model is not favored for $\mathrm{Sgr} \mathrm{A}^{*}$.

However, the approximation that the wind does not radiate may be an over-simplification. For example, the part of the wind originating from the supersonic region of the accretion disk will possibly be shocked when it is ejected out of the disk. Thus it would reach very high temperatures and its radiation could not be neglected. In this sense, the wind within the sonic radius will present itself as radiative, outflowing plasma - i.e., like the plasma jets typically observed in AGN. The model would then possibly become similar to our jet-disk model presented below.

\section{Jet-ADAF model for Sgr A*}

The idea of combining a jet and an ADAF was proposed by Falcke (1999) and Donea et al. (1999). Yuan (2000) first worked this out in detail and calculated the spectrum of the jet-ADAF system for Sgr A* and some nearby elliptical galaxies. There is only scant direct observational evidence for the existence of a jet in $\mathrm{Sgr} \mathrm{A}^{*}$, from the nearsimultaneous VLBA measurements by Lo et al. (1998). They found that the intrinsic source structure at $43 \mathrm{GHz}$ is elongated along an essentially north-south direction, with an axial ratio of less than 0.3 . However, it is interesting to note that the nearby spiral galaxy M81 has a very similar radio core and similar unusual polarization features as Sgr A* (Bietenholz et al. 2000; Brunthaler et al. 2001). In this source, a jet was clearly observed after many VLBI observations, with the length of the jet being only $\sim 400 \mathrm{AU}$ at $43 \mathrm{GHz}$ (Bietenholz et al. 2000). If we consider M81 
to be a scaled-up version of $\mathrm{Sgr} \mathrm{A}^{*}$, as suggested by their similarity, there could well exist a jet in Sgr A* as well. Of course, the jet in $\mathrm{Sgr} \mathrm{A}^{*}$ would be less powerful and hence smaller, making it difficult to detect because of the strong scattering of radio waves within the Galaxy. More generally, jets seem to be symbiotic with accretion disks (Falcke \& Biermann 1995; Livio 1999) and they are found in basically all kinds of accretion powered systems. In this sense the model presented here may be quite general.

The picture of our jet-disk model presented here is as follows. The accretion disk is described by an ADAF. In the innermost region, $r<r_{0}$, where parameter $r_{0}$ is the jet location, a fraction $q_{\mathrm{m}}$ of the accretion flow is ejected out of the disk and forms a jet. Since in our model $r_{0}$ is very small $\left(r_{0} \approx 2 R_{\mathrm{s}}\right.$, within the sonic point of the accretion disk), the radial velocity of the accretion flow is supersonic at this small radius (the Mach Number is $\sim 2-3$ ). Therefore, when the supersonic accretion flow is transferred from the disk into the jet, which is normal to the disk, the plasma will be shocked before entering into the jet. The shocked gas passes through a nozzle where it becomes supersonic. Then it is accelerated along the jet axis through the gas pressure gradient force (the gravitational force is ignored since its effect is rather small in the supersonic regime far away from the black hole) and expands sideways with its initial sound speed. Given the initial physical states of the plasma at the sonic point (top of the nozzle), we can solve for all the quantities as a function of distance from the nozzle, and after calculating the density and the strength of the magnetic field, we can calculate the radiation of the jet (Falcke \& Markoff 2000).

If, however, there exists a possibility that a substantial fraction of the accretion flow can be transferred into the jet directly without being shocked (e.g., the accretion flow outside of the sonic point also goes into the jet), we could also envisage a mixture of a relatively cold (unshocked, $\sim 10^{10} \mathrm{~K}$ in the innermost region of ADAF) and hot (shocked, $\sim 10^{11} \mathrm{~K}$, see below for this value) electrons in the jet. Since the energy transfer between two species of particles is inefficient due to the infrequent collision between them, this kind of mixture could last for a long distance along the jet. For an emission model we can ignore this possibility, because the implied radiation from the cold unshocked plasma should be much less than the dashed line in Fig. 3 and its contribution to the overall spectrum can be neglected. On the other hand, such a mixture of hot and cold electrons may be needed when considering the circular polarization of Sgr A* (Beckert \& Falcke 2002). This might increase the coupling constant between jet and disk.

The exact physics of the nozzle are difficult to model since we are at present unclear as to the physical mechanism of jet formation. In this paper we treat the nozzle only phenomenologically when calculating its spectrum. We simply assume that it consists of a series of cylinders with the same electron temperature but linearly decreasing density (increasing velocity) from bottom to top. The velocity of the gas at the base of the nozzle is assumed to be $1 / 5$ of that at the top of the nozzle where it reaches sound speed. The emission is not very sensitive to the exact value of the initial nozzle speed. The main radiation mechanisms are synchrotron emission and its Comptonization. The parameters describing the jet include radius and height of the nozzle, $r_{0}$ and $z_{0}$, electron temperature $T_{\mathrm{e}}$, electron number density $n_{\mathrm{e}}$, the strength of the magnetic field $B$ at the top of nozzle, and the angle between the jet axis and the line of sight $\theta$.

All above are free parameters in the original jet model (but most of them have obvious physical constraints to their range of values, see Falcke \& Markoff 2000). But here in our coupled jet-disk system, more constraints are required so that the jet parameters are consistent with the underlying accretion disk. $T_{\mathrm{e}}$ is calculated self-consistently as follows. When some accretion gas passes through the shock and enters into the jet, the ordered kinetic energy in the pre-shock gas will be converted into thermal energy in the shock front. Neglecting the effects of the magnetic field on the shock jump condition (we will check the rationality of this approximation later), we calculate the electron temperature of the post-shock plasma by the following Rankine-Hugoniot relations, namely the conservation of flux of mass, momentum, and energy. Written in the conventional notation, they are

$$
\begin{aligned}
& {[\rho v]=0,} \\
& {\left[P+\rho v^{2}\right]=0} \\
& {\left[\frac{1}{2} v^{2}+\frac{2}{5} \frac{P}{\rho}\right]=0}
\end{aligned}
$$

respectively. To obtain $T_{\mathrm{e}}$ immediately after the shock, we still need the ratio between the ion and electron temperatures, $\xi$. On the one hand, shock heating may favor the ions rather than electrons, as isotropization of the bulk flow velocities will give to each species a thermal energy proportional to its mass. On the other hand, Coulomb collisions, and maybe collisionless processes also, will bring about equilibration between ions and electrons temperatures. Determining the value of $\xi$ is a difficult task (see Laming 2000 for a recent review). In the context of supernova remnant shocks, Cargill \& Papadopoulos (1988) predict $\xi=5$ from a numerical simulation, while Laming et al. (1996) derive $\xi=20$ from their fit to observations. We set $\xi=10$ in our model.

For a given shock location $r_{0}$, we first solve the radiation-hydrodynamical equations describing the underlying ADAF under selected parameters and outer boundary conditions to obtain the pre-shock physical quantities at $r_{0}$. We then substitute them in the above shock relations to get the post-shock values. Thus we obtain the electron temperature $T_{\mathrm{e}}$ in the nozzle. Therefore $T_{\mathrm{e}}$ is not a free parameter in our model, we can change its value only through changing the parameters and outer boundary conditions of the underlying accretion disk. We use the same "magnetic parameter" $\beta$ as in the ADAF to describe the ratio between the gas pressure and total pressure in 


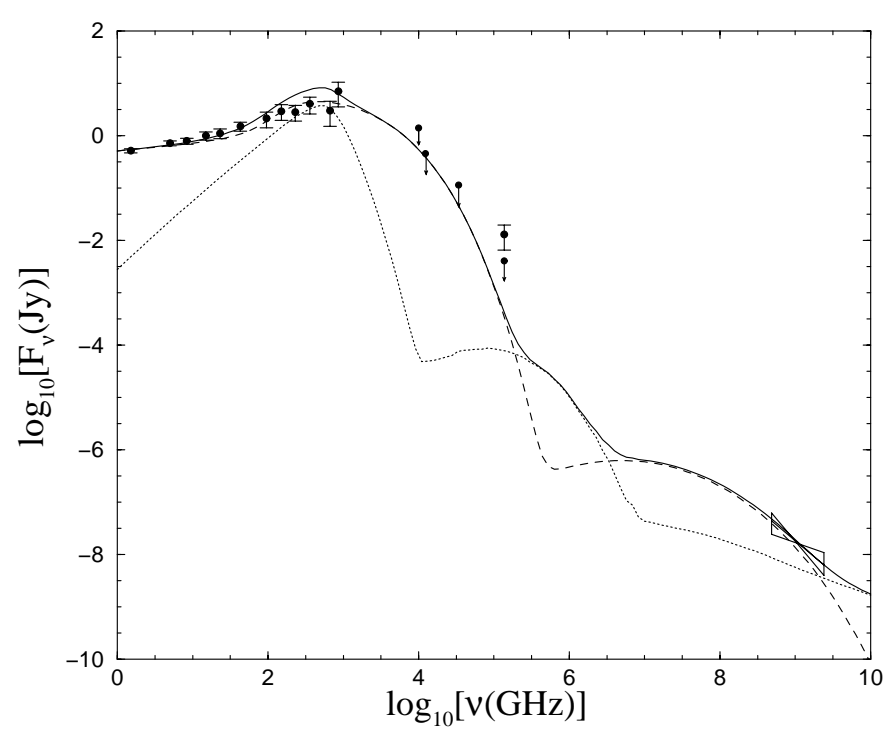

Fig. 3. The jet-disk spectral model for Sgr A*. The dotted line is for the ADAF contribution, the dashed line is for the jet emission, and the solid line shows their sum. See text for details.

the nozzle to obtain the value of $B$ if temperature and density are known. This means that $B$ is no longer a free parameter, either. The density, $n_{\mathrm{e}}$, in the jet follows from the coupling constant $q_{\mathrm{m}}$ in the jet-disk symbiosis model (Falcke et al. 1993). This is defined as the ratio between mass loss in the jet and accretion rate outside $r_{0}$. For jets, $q_{\mathrm{m}}$ is typically a few percent and we require $q_{\mathrm{m}} \ll 1$.

Our best spectral fit is presented by the solid line in Fig. 3. The dashed line denotes the emergent spectrum from the jet, and the dotted line is from the underlying disk (ADAF). The solid line is their sum. For ADAFs, the parameters are $\dot{M}=8.8 \times 10^{-7} M_{\odot} \mathrm{yr}^{-1}, \alpha=0.1$, $\beta=0.95$ and $\delta=10^{-3}$. The outer boundary conditions are $T_{\mathrm{i}} \approx T_{\mathrm{e}}=8 \times 10^{6} \mathrm{~K}, \Omega_{\text {out }}=0.27 \Omega_{\text {Kepler }}$ at $10^{5} R_{\mathrm{s}}$. The parameters for the jet are $r_{0}=1.7 R_{\mathrm{s}}, z_{0}=3.5 r_{0}$, $q_{\mathrm{m}}=0.5 \%$ and $\theta=35^{\circ}$, the "calculated parameters" are $B=23 \mathrm{G}, T_{\mathrm{e}}=2.1 \times 10^{11} \mathrm{~K}$, and $n_{\mathrm{e}}=2.4 \times 10^{6}$. The mass loss rate in the jet is $\dot{M}_{\text {jet }}=\pi r_{0}^{2} c_{\mathrm{s}} n_{\mathrm{e}} m_{\mathrm{p}}=$ $4.3 \times 10^{-9} M_{\odot} \mathrm{yr}^{-1}$, i.e. $0.5 \%$ of the accretion rate. For $\beta=0.95$ and shock location $r_{0}=1.7 R_{\mathrm{s}}$, the Alfvén Mach number $M_{A} \equiv v / v_{A 1}=\left(v / c_{\mathrm{S}}\right)\left(4 \pi \gamma p / B^{2}\right)^{1 / 2} \approx 10>6$, here $v_{A 1} \equiv B_{1} /\left(4 \pi \rho_{1}\right)^{1 / 2}$ is the pre-shock Alfvén speed, the magnetic effects are weak in the shock transition condition, so our hydrodynamic approximation to the shock transition condition, Eqs. (1)-(3) above, is justified (Draine \& McKee 1993).

This model fits the spectrum over the whole range of frequencies from radio to the X-ray quite well. The submm bump is slightly over-predicted, but it is acceptable considering the variability of the data in this band (Melia \& Falcke 2001) and the uncertainty of the model. The lowfrequency radio emission is mainly contributed by the jet outside the nozzle. The contribution from the ADAF is rather weak and can be neglected. The submm bump is the sum of the synchrotron radiation from both the ADAF and the nozzle of the jet. We note that the emission from the nozzle is much weaker than in the ADIOS with $\delta=1$ presented in the last section (solid line in Fig. 2) although the electron temperatures are both $\gtrsim 10^{11} \mathrm{~K}$. Such a difference is not surprising considering the much smaller spatial scale of the nozzle, $r_{0}=1.7 R_{\mathrm{s}}$, while in that case, there is a larger radial range with high temperature. In this sense, an abrupt increase in the temperature profile is necessary to model the spectrum. This is naturally satisfied in our jet-disk model by the formation of a jet. If instead the nozzle in our model is replaced by a similar high-temperature component such as the inner region of a disk, since the temperature profile of the disk is in general smooth, the radial range of this high-temperature component would be considerable. In this case, we expect that the model would greatly over-predict the submm flux, and the low-frequency radio spectrum is still hard to explain, as indicated by our calculation for the ADIOS model with high $\delta$ in Sect. 3. This is the reason why in Melia et al. (2001)'s model the authors require an accretion disk as small as $\sim 5 R_{\mathrm{s}}$.

The X-rays are mainly produced in the nozzle by SSC, although bremsstrahlung from the ADAF also contributes to some degree. The predicted X-ray spectrum is the sum of the very soft SSC from the nozzle and the relatively flatter bremsstrahlung spectrum from the ADAF, which is in very good agreement with the Chandra data, almost identical to the best fit of Baganoff et al. (2001b). The fit is also much better than that of the ADIOS with $\delta=1$ in the last section. In both cases, the X-ray emission is the sum of bremsstrahlung and SSC, but in the present case, bremsstrahlung produces a much flatter spectrum than in the case of an ADIOS due to the absence of a strong wind. Because of the contribution of SSC from the jet, the variability timescale of X-rays should be short, $t \approx r_{0} / v_{\text {jet }} \approx 10 \mathrm{~min}$. This is consistent with the $\sim 1$ hour variability timescale determined in the quiescent state and is in excellent agreement with the $600 \mathrm{~s}$ variability timescale detected in the flare state. We show that it is the variability of the flux from the nozzle that causes the huge-amplitude flare (Markoff et al. 2001b). On the other hand, since the bremsstrahlung radiation from the ADAF also contributes partly to the X-ray spectrum, this could explain the possibly detected extended source with $\sim 10^{5} R_{\mathrm{s}}\left(\approx 1^{\prime \prime}\right)$, the $6.7 \mathrm{keV} \mathrm{K} \alpha$ emission line, and steady $\mathrm{X}$-ray flux on $\sim$ one year timescale, as we stated in Sect. 2.

We note that the above nozzle parameters, temperature, spatial size and density, are very close to the "second component" in the model of Beckert \& Duschl (1997), which is also responsible for the submm bump of $\operatorname{Sgr} \mathrm{A}^{*}$. These parameters seem to be the best ones to fit the submm bump. It is interesting that the nozzle with these parameters will "evolve" naturally into a jet whose emission can well reproduce the low-frequency radio spectrum of $\mathrm{Sgr} \mathrm{A}^{*}$, and the Comptonization of its synchrotron emission can produce a very soft X-ray spectrum which can fit the Chandra data excellently. In fact, to make the upscattered submm bump extend to the Chandra band, an 
electron temperature as high as $10^{11} \mathrm{~K}$ is needed. The peak frequency of this bump is $\sim 10^{12} \mathrm{~Hz}$. To up-scatter it to the X-ray band, $\nu \sim 10^{16} \mathrm{~Hz}$, the electron Lorentz factor must satisfy $4 \gamma_{\mathrm{e}}^{2} \approx 10^{16} / 10^{12} \approx 10^{4}$. This corresponds to a temperature of $T \approx \frac{1}{k} \frac{\gamma_{\mathrm{e}}}{3.5} m_{\mathrm{e}} c^{2} \approx 10^{11} \mathrm{~K}$. This value is about 10 times higher than the highest temperature that a canonical ADAF can reach in its innermost region, but is naturally reached when some fraction of accretion matter is shocked ${ }^{3}$. In addition to a high temperature, the spatial size of the dominant emission medium must be small, otherwise the model will over-predict the high-frequency radio flux as in ADIOS with high $\delta$ (the solid line in Fig. 2). This is also easily satisfied in the jet model by requiring a small $r_{0}$. In addition to the above parameters, a truncated (no hard tail) electron energy distribution is also required in the model, otherwise the synchrotron emission will extend above the observed IR flux upper limit. Beckert \& Duschl (1997) simply assume a mono-energetic distribution. In our model, a relativistic thermal distribution, which is highly peaked at $\gamma \approx 3.5 \frac{k T}{m_{\mathrm{e}} c^{2}}$, is a natural result of shock heating (e.g. Drury 1983) since the Mach number is not very large in our case, $\sim 2-3$.

The mass accretion rate of the ADAF in our model, $8.8 \times 10^{-7} M_{\odot} \mathrm{yr}^{-1}$, is only marginally smaller than the lower limit of Baganoff et al. (2001a) estimate of $1 \times 10^{-6} M_{\odot} \mathrm{yr}^{-1}$. If we used a higher accretion rate, we would obviously slightly over-predict the flux at the submm bump band because of the higher flux of the synchrotron emission from the ADAF.

There are various ways to further evolve the model. One is to introduce global winds in the ADAF. The X-ray radiation from the disk would be almost unaffected but the radio emission from the disk would be greatly decreased because of the great decrease in density close to the black hole (Quataert \& Narayan 1999). But the wind cannot be too strong, otherwise the X-ray spectrum would be too soft, as we argued in the case of ADIOS model with $\delta \sim 1$. Another modification is to assume that the accretion disk is radiatively truncated within $r_{0}$, the radius of the jet formation (Yuan 2000). The physical reason for the truncation is that to form a jet, some amount of energy is needed. If we assume this energy comes from the underlying disk, the disk will be left cold within $r_{0}$ because of the energy extraction (Blandford \& Payne 1982). This will greatly suppress the synchrotron emission due to the very sensitive dependence of synchrotron radiation on the temperature.

\section{Summary and discussion}

Recent Chandra X-ray observations put new constraints on the theoretical models of Sgr A*. The spectrum is very soft, the flux is rapidly variable and the source is extended. In this paper we consider three different models to explain

\footnotetext{
${ }^{3}$ We note in this context that Falcke (1996b) and Beckert \& Duschl (1997) demanded that $T \approx 10^{11} \mathrm{~K}$ based on the Sgr A* spectrum alone.
}

the observational results of Sgr A*. We find that an ADAF model can give a marginally satisfactory interpretation to the Chandra spectrum and the rapid X-ray variability. But our best fit is still not good for the radio spectrum in the sense that it over-predicts the high-frequency radio by a factor of $2-3$ and significantly under-predicts the low-frequency radio. We then consider the possibility of strong winds from ADAFs, i.e., an ADIOS model. If the winds are non-radiative and viscous dissipation in the accretion flow mainly heats ions, as generally assumed in the literature, this model can fit the spectrum ranging from submm bump to X-ray quite well. However, it is hard to explain the rapid X-ray variability since in this model bremsstrahlung is the sole contributor at X-ray band. If we assume that most of the viscous dissipation preferentially heats electrons, a rapidly variable X-ray spectrum is expected since in this case the X-ray emission is dominated by SSC. But in this case the model over-predicts the radio flux above $\sim 100 \mathrm{GHz}$ by a factor of $4-6$, and the predicted X-ray spectrum is much steeper than the best fit of the Chandra observations.

An excellent fit to all the data including low-frequency radio can be obtained with a coupled jet-disk model. In this model, the accretion disk is described by an ADAF. In the innermost region of the $\mathrm{ADAF}, \sim 2 R_{\mathrm{s}}$, some fraction $q_{\mathrm{m}}(\sim 0.5 \%$ if any cold jet component is neglected. See our discussion in Sect. 4 for the possibility of a cold jet component) of the accretion flow is ejected out of the ADAF and transferred into the jet. In this process, a shock occurs because the accretion flow is radially supersonic before the shock. After the shock the temperature of electrons in the nozzle (the base of the jet) reaches about $2 \times 10^{11} \mathrm{~K}$. In this case, the synchrotron emission in the nozzle largely dominates the submm bump, and its Comptonization dominates the quiescent X-ray spectrum in $\mathrm{Sgr} \mathrm{A}^{*}$. The X-ray spectrum is soft and the variability timescale is short. Out of the nozzle, the jet gas expands freely outward under the force of the gas pressure gradient of gas pressure. Furthermore its self-absorbed synchrotron radiation gives a good fit to the low-frequency radio spectrum of Sgr $\mathrm{A}^{*}$ which is hard to explain in ADAF models. The model is completely self-consistent.

The jet in our model produces a slightly inverted radio spectrum, as can be understood from the canonical model of Blandford \& Königl (1979), with modifications as in Falcke (1996a). In the absence of a shock acceleration region in the highly-supersonic outer region of the jet, the particles retain the highly-peaked relativistic Maxwellian energy distribution which is attained by shock heating occurring when the radial supersonic accretion flow is transferred into the vertical direction. On the other hand, the electrons in AGN jets typically seem to have a powerlaw high-energy tail after shock acceleration in jets, since the Mach number in jets is very high (Drury 1983). In that case, a corresponding optically thin power-law spectrum at IR/optical frequencies is generally expected, as is seen in many AGN and perhaps even X-ray binary jets (e.g., Markoff et al. 2001a). In the case of Sgr A*, the 
absence of an optically-thin power-law indicates that, for some unknown reason, such high Mach number shocks do not occur. If they would occur under certain conditions, we should still see an inverted radio spectrum, but we would also expect some kind of hard power-law emission at higher frequencies (mid-IR to X-rays).

In addition to the observations we mention in the present paper, there are also constraints to the model through the frequency-size relationship obtained from VLBI observations (Rogers et al. 1994; Krichbaum et al. 1998; Lo et al. 1998). The jet-disk model can fit this well as shown in Falcke \& Markoff (2000).

We therefore conclude that it is possible to present a consistent picture of the emission processes associated with the central black hole in our Galaxy by combining the three basic astrophysical ingredients that have been discussed in recent years: Bondi-Hoyle accretion from the immediate environment, optically thin accretion through an ADAF, and energy extraction and visible emission by a plasma jet. Our jet-ADAF model predicts a closely correlated variability among sub-millimeter, IR, and X-ray. More broad-band observations and monitoring at various wavebands (radio, IR, X-rays) will help to judge whether it will be possible to establish a standard model invoking those elements for $\operatorname{Sgr} \mathrm{A}^{*}$ in the near future. For example, more precise determination of the IR flux will help to further discriminate between the jet-ADAF model and the pure ADAF model since the former predicts higher IR flux than the latter. This will also be crucial for understanding the activity in low-power black holes in general.

Acknowledgements. We are grateful to Peter Biermann for discussions on shock physics. F.Y. thanks the partial support from China 973 Project under NKBRSF G19990754.

\section{References}

Abramowicz, M. A. 1998, in Theory of Black Hole Accretion Disks, ed. M. A. Abramowicz, G. Björnsson, \& J. E. Pringle (Cambridge University Press)

Abramowicz, M. A., Chen, X., Kato, S., Lasota, J.-P., \& Regev, O. 1995, ApJ, 438, L37

Abramowicz, M. A, \& Zurek, W. H. 1981, ApJ, 246, 314

Aitken, D. K., Greaves, J. S., Chyrsostomou, A., et al. 2000, ApJ, 534, L173

Backer, D. C., \& Sramek, R. A. 1999, ApJ, 524, 805

Baganoff, F. K., Maeda, Y., Morris, M., et al. 2001a, ApJ, in press [astro-ph/0102151]

Baganoff, F. K., Bautz, M. W., Brandt, W. N., et al. 2001b, Nature, 413, 45

Beckert, T., \& Duschl, W. J. 1997, A\&A, 328, 95

Beckert, T., \& Falcke, H. 2002, A\&A, submitted [astro-ph/0112398]

Bietenholz, M. F., Bartel, N., \& Rupen, M. P. 2000, ApJ, 532, 895

Bisnovatyi-Kogan, G. S., \& Lovelace, R. V. E. 1997, ApJ, 486, L43

Bisnovatyi-Kogan, G. S., \& Lovelace, R. V. E. 2000, ApJ, 529, 978

Blandford, R. D., \& Begelman, M. C. 1999, MNRAS, 303, L1
Blandford, R. D., \& Königl, A. 1979, MNRAS, 232, 34

Blandford, R. D., \& Payne, D. G. 1982, MNRAS, 199, 883

Blackman, E. 1999, MNRAS, 302, 723

Bower, G. C., Falcke, H., \& Backer, D. C. 1999, ApJ, 523, L29

Brunthaler, A., Bower, G. C., Falcke, H., \& Melon, R. R. 2001, ApJ, submitted

Cargill, P. J., \& Papadopoulos, K. 1988, ApJ, 329, L29

Chen, X., Abramowicz, M. A., Lasota, J.-P., Narayan, R., \& Yi, I. 1995, ApJ, 443, L61

Chen, X., Abramowicz, M. A., \& Lasota, J.-P. 1997, ApJ, 476, 61

Coker, R. F. 2001, A\&A, 375, L18

Coker, R. F., \& Melia, F. 1997, ApJ, 488, L149

Das, T. K., \& Chakrabarti, S. K. 1999, Class Quant Grav, 16, 3879 [astro-ph/9912493]

Di Matteo, T., Quataert, Q., Allen, S. W., Narayan, R., \& Fabian, A. C. 2000, MNRAS, 311, 507

Donea, A. C., Falcke, H., \& Biermann, P. L. 1999, in The Central Parsecs of the Galaxy, ed. H. Falcke, A. Cotera, W. J. Duschl, F. Melia, \& M. J. Rieke, ASP Conf. Ser., 186,162

Drain, B. T., \& McKee, C. F. 1993, ARA\&A, 31, 373

Drury, L. 1983, Rep. Prog. Phys., 46, 973

Eckart, A., \& Genzel, R. 1996, Nature, 383, 415

Falcke, H. 1996a, ApJ, 464, L67

Falcke, H. 1996b, in Unsolved Problems of the Milky Way, IAU Symp., 169, 169, 169

Falcke, H. 1999, in The Central Parsecs of the Galaxy, ed. H. Falcke, A. Cotera, W. J. Duschl, F. Melia, \& M. J. Rieke, ASP Conf. Ser, 186, 113

Falcke, H., \& Biermann, P. L. 1995, A\&A, 293, 665

Falcke, H., \& Biermann, P. L. 1999, A\&A, 342, 49

Falcke, H., Goss, W. M., Matsuo, H., Teuben, P., Zhao, J. H., \& Zylka, R. 1998, ApJ, 499, 731

Falcke, H., \& Heinrich, O. 1994, A\&A, 292, 430

Falcke, H., Mannheim, K., \& Biermann, P. L. 1993, A\&A, 278, L1

Falcke, H., \& Markoff, S. 2000, A\&A, 362, 113

Falcke, H., \& Melia, F. 1997, ApJ, 479, 740

Falcke, H., Nagar, N. M., Wilson, A. S., \& Ulvestad, J. S. 2000, ApJ, 542, 197

Ghez, A. M., Klein, B. L., Morris, M., \& Becklin, E. E. 1998, ApJ, 509, 678

Gruzinov, A. 1998, ApJ, 501, 787

Haller, J. W., Rieke, M. J., Rieke, G. H., et al. 1996, ApJ, 468, 955

Ichimaru, S. 1977, ApJ, 214, 840

Krichbaum, T. P., Graham, D. A., Witzel, A., et al. 1998, A\&A, 335, L106

Laming, J. M. 2000, ApJS, 127, 409

Laming, J. M., Raymond, J. C., McLaughlin, B. M., \& Blair, W. P. 1996, ApJ, 472, 267

Livio, M. 1999, Phys. Rep., 311, 225

Lo, K. L., Shen, Z. Q., Zhao, J. H., \& Ho, P. T. P. 1998, ApJ, 508, L61

Mahadevan, R. 1997, ApJ, 477, 585

Mahadevan, R. 1998, Nature, 394, 651

Manmoto, T., Mineshige, S., \& Kusunose, M. 1997, ApJ, 489, 791

Markoff, S., Falcke, H., \& Fender, R. 2001a, A\&A, 372, L25

Markoff, S., Falcke, H., Yuan, F., \& Biermann, P. 2001b, A\&A, 379, L13

Melia, F. 1992, ApJ, 387, L25 
Melia, F. 1994, ApJ, 426, 577

Melia, F., \& Falcke, H. 2001, ARA\&A, 39, 309

Melia, F., Liu, S., \& Coker, R. 2001, ApJ, 553, 146

Menten, K. M., Reid, M. J., Eckart, A., \& Genzel, R. 1997, ApJ, 475, L111

Mezger, P. G., Duschl, W. J., \& Zylka, R. 1996, A\&AR, 7, 289

Nakamura, K. E., Kusunose, M., Matsumoto, R., \& Kato, S. 1997, PASJ, 49, 503

Narayan, R., Mahadevan, R., Grindly, J. E., Popham, R., \& Gammie, C. 1998, ApJ, 492, 554

Narayan, R., \& Raymond, J. 1999, ApJ, 515, L69

Narayan, R., \& Yi, I. 1994, ApJ, 428, L13

Narayan, R., \& Yi, I. 1995, ApJ, 444, 231

Narayan, R., Yi, I., \& Mahadevan, R. 1995, Nature, 374, 623

Narayan, R., Kato, S., \& Honma, F. 1997, ApJ, 476, 49

Özel, F., Psaltis, D., \& Narayan, R. 2000, ApJ, 541, 234

Paczyński, B., \& Wiita, P. J. 1980, A\&A, 88, 23

Predehl, P., \& Trümper, J. 1994, A\&A, 290, L29
Quataert, E., \& Gruzinov, A. 1999, ApJ, 520, 248

Quataert, E., \& Gruzinov, A. 2000, ApJ, 545, 842

Quataert, E., \& Narayan, R. 1999, ApJ, 520, 298

Rees, M. J., Begelman, M. C., Blandford, R. D., \& Phinney, E. S. 1982, Nature, 295, 17

Reid, M. J., Readhead, A. C. S., Vermeulen, R. C., \& Treuhaft, R. N., 1999, ApJ, 524, 816

Reynolds, S. P., \& McKee, C. F. 1980, ApJ, 239, 893

Rogers, A. E. E., Doeleman, S., Wright, M. C. H., et al. 1994, ApJ, 434, L59

Serabyn, E., Carlstrom, J., Lay, O., et al. 1997, ApJ, 490, L77

Xu, G., \& Chen, X. 1997, ApJ, 489, L29

Yuan, F. 1999, ApJ, 521, L55

Yuan, F. 2000, MNRAS, 319, 1178

Yuan, F., Peng, Q. H., Lu, J. F., \& Wang, J. M. 2000, ApJ, 537, 236

Zylka, R., Mezger, P. G., \& Lesch, H. 1992, A\&A, 261, 119 